\title{
Brisbane Adolescent Twin Study: Outline of study methods and research projects
}

\author{
MARGARET J. WRIGHT, \& NICHOLAS G. MARTIN \\ Genetic Epidemiology Laboratory, Queensland Institute of Medical Research, Brisbane, Queensland, Australia
}

\begin{abstract}
An ongoing program at the Queensland Institute of Medical Research (QIMR) uses a resource of adolescent twins (2720 individuals) and their singleton siblings (1179), constituting 1324 families. A large number ( $>500$ families) have participated in two main studies (the mole and the cognition studies) with the aim of identifying genetic polymorphisms associated with moliness and cognitive function respectively, as well as several other projects that run in parallel with these studies. Most recently 950 families were invited to participate in a mail and phone study assessing reading ability, taste and smell sensitivity, and health and wellbeing. Although the program measures a broad range of phenotypes, the common focus of all studies is to reveal the genetic architecture underlying the trait, and thereby facilitate the search for quantitative trait loci. The present paper provides an overview of the studies, and highlights the significance of the resource, as well as some of the unique aspects of twin research.
\end{abstract}

Twin studies have been used for centuries to investigate the heritability of a particular trait, and provide a method for investigating and partitioning the relative contributions of genes and the environment to phenotypic variability in all human traits. The classical "twin design" capitalises on the fact that identical, or monozygotic (MZ), twin pairs are similar because they share $100 \%$ of their genes and, when reared together, $100 \%$ of their shared (common) environment, with differences between them attributed to their nonshared (unique) environment. Nonidentical twins, or dizygotic (DZ) twins, on the other hand, are similar because they share on average only $50 \%$ of their genes and, when reared together, $100 \%$ of their shared (common) environment. Assuming that common environmental factors promote resemblance equally in $\mathrm{MZ}$ and $\mathrm{DZ}$ twin pairs (Plomin, De Fries, McClearn, \& Rutter, 1997), greater similarity of $\mathrm{MZ}$ than $\mathrm{DZ}$ pairs is attributed to genetic factors. However, if the degree of similarity is the same for $\mathrm{MZ}$ and $\mathrm{DZ}$ twins this suggests the trait is influenced by common environmental factors.

The key statistical tool in twin research is structural equation modelling (SEM), also referred to as path analysis, in which genetic and environmental effects are modelled as latent variables on the phenotypic difference of a measured trait between individuals. The contributions of the latent variables are estimated as regression coefficients in the linear regression of the measured trait on the latent variable. Modern twin analyses extend beyond the classical twin study for estimating the heritability of a trait, and allow the simultaneous modelling of two or more (dependent) variables in one analysis to investigate the contribution of genetic and environmental factors to the covariation among traits. This allows one to examine to what extent common genes pleiotropically influence multiple traits, and what extent there are specific genetic effects. For a detailed explanation of the various methods applicable to twin samples, the reader is referred to the following: Boomsma, Busjahn, and Peltonen, 2002; Evans, Gillespie, and Martin, 2002; Martin, Boomsma, and Machin, 1997; Neale and Cardon, 1992. More recently a twin pair-based approach (cf. the more usual extended pedigree or sibpair design) to linkage and association studies to map quantitative trait loci (QTL: a chromosomal region that contributes to variability in a complex quantitative trait)

Correspondence: M. J. Wright, Genetic Epidemiology Laboratory, Queensland Institute of Medical Research, Post Office, Royal Brisbane Hospital, Qld 4029, Australia. Tel.: +6173362 0225. Fax: +61733620101. E-mail: margieW@qimr.edu.au

ISSN 0004-9530 print/ISSN 1742-9536 online (C) The Australian Psychological Society Ltd 
has been developed with notable success (Knoblauch et al., 2000).

The purpose of this paper is to provide an overview of the adolescent twin studies (Table I) that are being undertaken in the Genetic Epidemiology Laboratory, at The Queensland Institute of Medical Research (QIMR) in Brisbane (information accessible at http:// www.genepi.studies.au). Although the studies are somewhat diverse, a common focus is to elucidate the genetic and environmental architecture underlying the phenotype, thereby facilitating the search for QTLs that influence these traits. The discovery of novel genes or novel variants of known genes that directly influence a trait may potentially lead to the isolation of a specific protein and/or possible regulating mechanism at the molecular level.

The research program is based upon an adolescent twin sample initially recruited in 1992, with new twins being added at various intervals, and referred to as the Brisbane Adolescent Twin Sample. The sample includes both $\mathrm{MZ}$ and $\mathrm{DZ}$ twin pairs, including opposite-sex twin pairs, and although the focus has been on the recruitment of twins, other multiples (triplets and quadruplets) and singleton siblings of twins/multiples are also included. The use of an extended twin design by including nontwin siblings greatly increases statistical power to detect genetic and shared environmental influences on a measured variable (Posthuma \& Boomsma, 2000). In addition, by adding a singleton sibling, $M Z$ pairs become informative for mapping genes using linkage and within-pair association analysis (by themselves they are not), and DZ twins become more than twice as informative. We have also included the parents of the twins, and although only minor demographic

Table I Timeline of studies

\begin{tabular}{|c|c|}
\hline 1992 & $\begin{array}{l}\text { - 12- and 14-year-olds mole study } \\
\text { Studies in parallel with mole study (12 and } 14 \text { years) } \\
\text { Personality } \\
\text { Haematology and immunology } \\
\text { Acne } \\
\text { Height and weight } \\
\text { Fingerprints } \\
\text { Serum biochemistry } \\
\text { }{ }^{\mathrm{b}}\end{array}$ \\
\hline 1996 & - 16-year-olds cognition study ${ }^{c}$ \\
\hline 2000 & $\begin{array}{l}\text { - Additional studies at age } 12 \text { and } 14 \\
\text { Binocular rivalry (age } 14 \text { ) } \\
\text { Psychological health (age } 12 \text { and } 14)^{\mathrm{d}}\end{array}$ \\
\hline 2002 & $\begin{array}{l}\text { - } 18+\text { year-olds pelvic floor Study (female subjects only) } \\
\text { - } 10-25 \text {-year-olds mail (and phone) study } \\
\text { - Additional study of handprints at } 12,14 \text { and } 16 \text { years }\end{array}$ \\
\hline
\end{tabular}

Note. ${ }^{a}$ Started 1993 (prints taken at one time-point; ${ }^{b}$ started 1994;

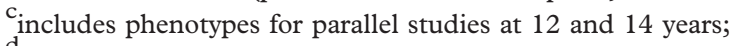

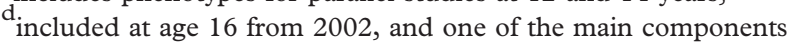
of the 2002 mail study; ${ }^{e}$ includes measures for acne, height and weight, haematology and immunology, and serum biochemistry. information is routinely collected from parents, a large majority of the parents have donated a blood sample and therefore are useful in error detection in linkage studies as well as haplotype (a region on a chromosome that is bounded by recombination sites such that genetic loci within a haplotypic region are usually inherited as a unit) determination.

At present there are three ongoing research studies that involve in-person assessment: the mole study, which began in 1992; the cognition (or memory, attention, and problem solving [MAPS]) study, which began in 1996; and the pelvic floor study (which is restricted to women) that began last year (2002). For all three studies data collection is labour intensive and time consuming, and thus expensive, and necessarily involves a smaller number of pairs than is usually available with studies in which phenotypes can be collected via questionnaire and at significantly lower cost. However, given the nature of the studies, the sample sizes thus far collected are considerable. In parallel with the mole, cognition, and pelvic floor studies, are several additional studies for which the phenotypes are collected at the same time, and most recently, a large mail and phone study was initiated. The objectives of these studies are briefly summarised below.

\section{Mole study}

The primary objective of the mole study is to identify major genes affecting moliness, pigmentation and other risk factors for melanoma. The presence of melanocytic naevi is the strongest-known risk factor for this disease (Swerdlow \& Green, 1987), and because the incidence of melanocytic naevi peaks around the age of puberty (Green \& Swerdlow, 1989), knowledge of naevus development in adolescents may further our understanding of the causes of malignant melanoma. The numbers of naevi (i.e., moles) as well as other risk factors for melanoma are collected from twins and siblings at 12 years and then again at 14 . The heritability of total naevus count turns out to be quite high, and the study is employing sibpair linkage and association analysis to examine the influence of genes involved in the susceptibility to and development of metastatic melanoma on the degree of moliness.

\section{Cognition study}

The cognition study follows on from the mole study in that many of the twins who participated in the mole study when they were 12 and 14 years old, participate in the cognition study when they are 16 years old. The cognition study is trying to identify some of the major genes associated with normal variation in cognitive ability. Two main cognitive 
domains are tapped (processing speed and working memory), with several performance/behavioural and electrophysiological indices, in addition to measures of general cognitive ability (i.e., psychometric IQ). Although there have been many studies that have examined the genetic influence on IQ, the extent of genetic influences on memory and processing speed is not well studied. The use of a range of measures in multiple domains enables multivariate analysis and this can increase the power of detecting QTLs. Moreover, statistical power to identify genes is thought to be maximised with the use of elementary or neurobiological traits (termed intermediate or endophenotypes) that correlate with the complex trait but are physiologically "closer" to the gene(s) (Almasy \& Blangero, 2001; Boomsma, Anokhin, \& de Geus, 1997). The cognition study (Wright, de Geus et al., 2001) is in collaboration with G. and L. Geffen, University of Queensland, and G. Smith, Department of Defence, Adelaide (previously University of Queensland). International collaborators include D. Boomsma and E. de Geus, Free University, Amsterdam, and J. Ando, Keio University, Tokyo.

\section{Pelvic Floor study}

The newly initiated pelvic floor study targets nulliparous female twin pairs, 18 years and over, and all of these twins participated in either or both the mole and cognition studies. Stress incontinence is a major health problem in older women (MacLennan, Taylor, Wilson, \& Wilson, 2000), and high pelvic organ mobility, measured by ultrasound, in young women has been shown to predict pelvic organ prolapse in later life (Dietz, Herbison, \& Clarke, 2001). The main aim of the pelvic floor study is therefore to estimate whether there is any genetic influence that accounts for the large individual variation in pelvic organ mobility. This study is in collaboration with P. Dietz, Royal Hospital for Women, Sydney.

Studies in parallel with the mole, cognition, and pelvic floor studies

There are several studies for which the phenotypes are collected at the same time as the mole, cognition and pelvic floor studies, at either one or multiple time-points (i.e., collected at 12,14,16, and some at $18+$ years). Some of these phenotypes involve physical measurement but minimal time to collect (e.g., height and weight, acne), or are easily assessed by questionnaire (e.g., personality, mental health) or require only a small sample of blood (e.g., haematological and immunological measures, and serum biochemistry) and are therefore measured at each time-point. Others need to be measured only once (e.g., fingerprints) or are more involved, requiring specialised equipment and considerable time, and are measured at one time-point (e.g., rivalry rate: a measure of the brain's switch rate). Early on when the mole study protocol was being developed, it was realised that having twins come in together meant that there was a need to occupy both of them, and that by using two research assistants rather than one, twice the amount of measurement could be done in the same time. In addition it provided the methodological advantage of having co-twins assessed independently. Rather than ask families only to come in for an hour, some after travelling a considerable distance, it was decided that this afforded the opportunity to collect multiple unrelated phenotypes at the same visit, and therefore accommodate several studies rather than only one. The studies necessarily include several different collaborators including I. Frazer, University of Queensland and Princess Alexandra Hospital (haematological and immunological study), J. Whitfield, Royal Prince Alfred Hospital, Sydney (serum biochemistry study), D. Loesch, LaTrobe University, Melbourne (dermatoglyphics: finger ridge count), J. Pettigrew, University of Queensland (binocular rivalry), and I. Hickie, University of Sydney, (mental health study). Data for two of the studies (the haematological and immunological study, and the personality studies) are currently the basis for two doctoral theses.

\section{Mail and phone study}

The mail and phone study targets twin pairs and their siblings aged from 10 to 25 years ( $>2400$ individuals), a large number of whom participated in the mole and/or cognition studies. As the name implies, it does not involve an in-person assessment in that data are collected by self-report and a telephone interview; data collection for this study was completed in November 2003. The study comprised various components, many of which are unrelated: mental health, leisure, personality, laterality, asthma, attitudes towards parenting, olfactory function, taste perception, and reading, but which were administered together for efficiency. While measures of personality and laterality, and more recently mental health, have been collected as part of the mole and cognition studies, the mail study provided the opportunity to expand and extend on these with a larger sample, and more refined and/or comprehensive measures. For example, at 12, 14 and 16 years, personality was assessed with the Junior Eysenck Personality Questionnaire, whereas in the mail study (those $17+$ years) it is assessed with the NEO Personality Inventory-Revised (NEO PI-R) (Costa \& McCrae, 1992). 
There are three main objectives of the mail and phone study. One is to examine the extent to which genetic variation influences mental health, and whether there are common familial factors that influence associations among indices of mental health, with leisure and/or personality, and academic achievement. Although many factors of an adolescent's development contribute to their overall mental health, leisure pursuits, which occupy as much as $40 \%$ of the waking time of an adolescent, may predict up to $24 \%$ of the variance in mental health (Passmore \& French, 2000), and several studies in both adult and university student samples have demonstrated a relationship between personality, mental health and educational outcome (e.g., Berlanga, Heinze, Torres, Apiquian, \& Caballero, 1999; Gershuny \& Sher, 1998).

The second objective is to look for genes accounting for the wide variation in reading ability/disability. Although reading is assessed in a limited way in the cognition study, in the mail and phone study a much more specific set of reading measures, with a particular emphasis on tapping diverse components of the reading processing (orthographic skill and phonological decoding), is employed. The study is a new collaborative effort with $M$. Coltheart, T. Bates, and A. Castles, Macquarie University, Sydney, who devised the reading tests, and is timely because recently there has been considerable success, primarily with a group in Oxford, in locating QTLs for several psychometric reading/language measures in families with at least one reading-impaired proband (Consortium, 2002; Fisher et al., 2001). The intention is to focus on these chromosomal regions for multivariate linkage analysis of reading ability, and to collaborate with the Oxford group in scanning these regions for potential candidate genes.

The third main objective is to examine the genetic variation of both taste and smell, and this is also a new collaboration with D. Reed and P. Breslin, Monell Chemical Senses Center, Philadelphia. While research has established the importance of genetic factors on taste perception (e.g., Guo \& Reed, 2001) and olfactory functioning (e.g., Finkel, Pedersen, \& Larsson, 2001; Segal, Topolski, Wilson, Brown, \& Araki, 1995), the goals of this study are to learn more about genetic variation in taste sensitivity for a range of different compounds (bitter and sweet), and odour detection and identification across odorants, and to identify the genes that account for some of this variation (e.g., Drayna et al., 2003). The study uses a range of tests and measures, some specifically designed by the Monell Center, in both modalities, and the determination of taste sensitivity and olfactory functioning in the same twin cohort adds considerable value to the collection by allowing multivariate study designs.
In the sections below we provide an outline of the recruitment and study methods: procedures, the assessment battery, the collection of DNA, and data management. We also describe the characteristics of the sample collected to date, highlight some of our published findings, and provide selected preliminary results of our ongoing analyses. The overall aim is to provide an appreciation of the resource that is available, the breadth and depth of the data collected, and the wide range of phenotypes that have thus far been assessed, and that are buttressed by several national and international collaborations.

\section{Methods}

\section{Recruitment of families with adolescent twins}

The majority of the twins and their nontwin siblings who participate in our adolescent studies were recruited through the primary and secondary schools in South East Queensland. In addition, a small number of twins has been recruited through word of mouth, or through the Australian Twin Registry (Hopper, 2002). Recruitment through the schools involved, in the first instance, seeking approval from the Department of Education to enlist the cooperation of primary and secondary school principals in identifying twins/multiples. A letter was then sent to each of the principals outlining the aims of the studies, together with several twin recruitment packs to be given out to twins enrolled at the school. The twin recruitment pack contained a letter to the twins and their parents providing information about the studies, directions on how to register, the details of the person who should be contacted for further information, a registration form, and a stamped addressed envelope. The first recruitment, which was specifically for the mole study, took place in 1992, with all (340 in total) primary school principals being contacted in the greater Brisbane region. In 1998, all (eighty-six State and eight-one private) secondary schools were approached and asked for help in identifying 12-18-year-old twins for the 16-yearolds cognition study. Primary schools were approached again in 2000, and at the time of writing all (530) primary schools are in the process of being approached. In addition, there were also some minor recruitment efforts in the intervening years.

If a family accepts the invitation to register, they fill out the registration form, providing contact details, names, date of birth, and zygosity of the twins, as well as the details of nontwin siblings and parents, and information on an alternate contact person who can assist with future address updates, and return the form in the stamped addressed envelope. On return of the registration form the personal information for each family member is logged into a demographic 
database. Each family member is assigned a unique 7-digit identification (ID) number and an "availability status" to identify "active" participants who may be approached for the various studies, and to identify those who can no longer (e.g., moved overseas), or no longer wish to participate.

Thus, the adolescent twin database is similar to other twin registries, in that it is volunteer based and individuals are unselected for their health or medical history, but it is somewhat unique in that the majority of twins are either adolescents or young adults, and that the database is regional rather than nationally based. Its regional nature is essential given that the initial aim was to provide a resource for studies requiring laboratory-based in-person assessment rather than that of the questionnaire type.

\section{General study procedures}

For both the mole study and cognition study, the general procedure is the same. Twins are contacted by letter close to their 12th and 14th (mole study) or 16th birthday (cognition study) and invited to participate. Shortly after, a research assistant telephones them to explain the purpose of the study, ascertains whether they are willing to participate, and schedules a time for testing. In addition, for the cognition study several screening questions regarding the status of the twins' health to identify possible medical factors that might affect their test performance (e.g., vision/hearing impairments, closed head injury, psychiatric disorder, recent illness, medication, recreational drugs) are asked of one of the parents.

For the mole study, twins attend together, with at least one of their parents accompanying them, along with any nontwin sibling (aged $10-18$ ) who agrees to participate, the average visit taking $2 \mathrm{hr}$. With two research assistants doing the assessments (detailed below), the time for each twin is approximately split between the counting of moles and carrying out of other physical measurements, and the completion of a questionnaire. In the beginning all twins were approached, but more recently, given that we have sufficient numbers of $\mathrm{MZ}$ twin pairs for estimating heritability and genetic modelling, MZ twins who have no singleton siblings have not been included because $M Z$ twins with no sibling are not informative for genetic linkage analysis.

Twins also attend together for the cognition study, with one research assistant testing one of the cotwins on a set of tasks while another research assistant tests the other co-twin on a different set of tasks. Half way through the $3 \frac{1}{2}$-hr testing session a break is given and the twins change over so that each of the twins gets tested on an identical test battery. Nontwin siblings are also invited to participate as close as possible to their 16th birthday, and are tested on an identical battery to the twins. Similarly for the pelvic floor study, female twins over the age of 18 were approached by letter and then followed up $2-3$ days later by a phone call. In this study around two thirds of the twins attended the testing session together, and a session takes approximately $35 \mathrm{~min}$ per participant.

For the mail and phone study, a twin study pack is sent to potential participants in the mail. Because this study does not involve in-person assessment, the target sample includes all twins and siblings in our demographic database that were identified as willing participants and aged 10-25 years, with the exception of $\mathrm{MZ}$ twin pairs with no singleton sibling, who are not informative for linkage. The approach pack contains a letter outlining the aims of the study, instructions on what to do, the name and phone number of the person to contact for more information, a consent form, and test materials and instructions (three components: questionnaire, psychophysical tests, reading test). The questionnaire and psychophysical tests are self-administered, and the reading test (the test is in a sealed envelope with the instruction "do not open until research interviewer calls") is administered over the phone by a research interviewer who contacts participants within 2 weeks of the twin study pack being mailed. Completed questionnaires, taste/smell score sheets, and signed consent forms are returned in a reply paid envelope.

\section{Assessment battery for each study}

Table II lists the assessment battery for the studies. The main phenotype for the mole study is naevus (mole) count. Naevi are counted at each body region (face, neck, back, arms and legs) and subclassified by size $(<2 \mathrm{~mm}, 2-5 \mathrm{~mm},>5 \mathrm{~mm}$ ), type (flat, raised, atypical) and site. Skin, hair and eye color, and degree of facial freckling are rated by a research nurse. Skin color is measured objectively by reflectance spectrophotometry. An objective measure of sun damage is also obtained by taking a silicon mould of the back of the hand, which is scored under a low-powered microscope on a 6-point rating scale. Detailed information is obtained on patterns of sun exposure using a standard "sun questionnaire" and the parent who accompanies the twins for testing is asked to complete a questionnaire with items on ancestry, family history of skin cancer and melano$\mathrm{ma}$, and patterns of sun exposure in the twins. The twins return at age 14 when the protocol is repeated, and between the visits at 12 and 14 years the twins are sent a diary to record their sun exposure (e.g., hours spent in sun, sunburns) over a 4-day weekend (Friday through Monday). 
Table II Assessment battery for each study

Mole study (ages 12 and 14)

- Mole count (flat, raised, atypical; count for each three categories )

- Skin, eye, hair colour (3-5-point scale)

- Skin reflectance (reflectometer; continuous)

- Freckling (4-point scale)

- Sun exposure (questionnaire, diary)

- Sun damage (silicon mould; 4-point scale)

Cognition study (age 16)

- Psychometric IQ (Multidimensional Aptitude Battery; 5 subtests, Weschler Adult Intelligence Scale - Revised Digit symbols sub-test)

- Reading (Cambridge Contexualised Reading Test Schonell Graded Word Fluency Test)

- Processing speed (inspection time, choice reaction time, P300 latency)

- Working memory (delayed response task performance, P300 amplitude, EEG slow wave)

- Resting EEG (delta, theta, alpha, beta)

- Academic achievement (Queensland Core Skills Test - year 12)

- Education (questionnaire)

- Handedness (Annett Handedness Questionnaire, 6 questions) ${ }^{\text {a }}$

Pelvic floor study (age 18+)

- Mobility of pelvic organs

- Joint elasticity

Parallel studies (ages $\left.12,14,16{\text { (also } 18^{\star} \text {, obtained once }}^{\star \star}\right)^{\mathrm{b}}$

- Personality (Junior Eysenck Personality Questionnaire)

- Haematology and immunology (blood cell measures (e.g., haemoglobin, blood cell count (red, white, platelet) and lymphocyte Analysis (e.g., monoclonal antibodies to CD3) ${ }^{\star}$

- Acne (face, chest, back; graded on 4-point scale) ${ }^{\star}$

- Height and weight*

- Fingerprints (dermatoglyphics: finger ridge count) ${ }^{\star \star}$

- Serum biochemistry (serum iron, ferritin, transferrin, transferrin receptor concentrations) ${ }^{\star}$

- Binocular rivalry (switch rate) ${ }^{\star \star}$

- Psychological health (SPHERE) ${ }^{\mathrm{c}}$

- Handprints (finger length ratio)

Mail and phone study (Age 10-25)

1. Health and wellbeing (questionnaire)

- Psychological health (SPHERE)

- Leisure (3 domains assessed: achievement, social, relaxation)

- Personality (NEO PI-R) - 17 + years only

- Social interactions

2. Taste and olfaction (psychophysical tests)

- Taste sensitivity to 6-n-propyl-2-thiouracil

- Taste sensitivity to bitter and sweet compounds (2 sets of 10 compounds)

- Brief Smell Identification Test (scratch \& sniff)

- National Geographic Smell Survey (scratch \& sniff)

3. Reading (phone interview)

- Reading and spelling tests of nonwords, regular words and irregular words a phonetic spelling test and a test of word meanings 4. Various (questionnaire)

- Laterality (handedness; 12 questions [Annett, 1970, foot and eye preference)

- Asthma, eczema, allergies

- Author recognition (number of authors identified from nonauthors)

- Demographics

- Attitudes to parenting $(17+$ only)

Note: $E E G=$ electroencephalogram; SPHERE $=$ Somatic and Psychological Health Report

${ }^{a}$ Handedness for parents of twins in the cognition study also obtained; handedness questionnaire administered at 12 and 14 from 2001 ; and included in full form (12 questions as opposed to 6) in mail study.

${ }^{\mathrm{b}}$ Questionnaire about ancestry and twins' birth (e.g., birth weight, placentation, delivery, etc.) administered at one time-point to parent.

${ }^{\mathrm{c}}$ Administered at 16 years from 2002, also major component of mail study.

${ }^{\star}$ Assessment also obtained at 18 years.

${ }^{\star \star}$ Assessment only obtained once.

The cognition study assessment battery includes psychometric measures of cognitive ability (five subtests of the Multidimensional Aptitude Battery (MAB) (Jackson, 1998) and one subtest (digit symbols) of the Weschler Adult Intelligence Scale
Revised (WAIS-R) (Wechsler, 1981), information processing measures (choice reaction time, CRT; inspection time, IT) (Luciano et al., 2001), reading ability (Contextualised Cambridge Reading test; CCRT) (Beardsall \& Huppert, 1994) a contextua- 
lised version of the National Adult Reading Test (NART), Schonell Graded Word Reading test (SGWRT), electrophysiological (P300 amplitude and latency, slow wave [SW] amplitude) and performance measures (accuracy) of working memory (Hansell et al., 2001; Wright, Hansell et al., 2001), and psychophysiological measures (electroencephalogram [EEG] spectral power). Twins/sibs also complete a questionnaire with respect to their grades at school, when they started school and if they had to repeat a year. In addition, a measure of academic achievement, the Queensland Core skills test (QCST), which the majority of Grade 12 Queensland students take in their final year at secondary school, is obtained from the Queensland Senior Secondary Schools Board.

The pelvic floor study assessment battery consists of a mobility measure of the pelvic organs (e.g., bladder), using ultrasound imaging. In addition, three measures of joint flexibility (mobility of finger joints, the elbow and the wrist) are obtained.

The test battery for the studies done in parallel with the mole, cognition and pelvic floor studies includes the assessment of personality (Junior Eysenck Personality Questionnaire) (Eysenck \& Eysenck, 1975), haematological (haemoglobin, mean corpuscular volume, haematocrit, blood cell counts [red, white, and platelet]) and immunological (e.g., CD4 + T-lymphocyte count, CD4 +/ $\mathrm{CD} 8$ + ratio) measures (Evans, Frazer, \& Martin, 1999), acne (rating on a 4-point scale), height and weight, dermatoglyphics (finger ridge count from fingerprints), serum biochemistry (serum iron, ferritin, transferrin, and transferrin receptor concentrations), interhemispheric switching (binocular rivalry switch rate) (Miller et al., 2000), psychological health (Somatic and Psychological Health Report: SPHERE) (Hadzi-Pavlovic et al., 1997) and handprints (finger length ratio). While some of the phenotypes, such as those of height and weight, and personality were in the original 12-year-olds mole study protocol in 1992, other measures have been incorporated at various stages, so that the sample size for some of the phenotypes (e.g., interhemispheric switching) is currently small (150 twin pairs). In addition, parents provide information regarding the twins' birth and ancestry, as well as their fingerprints, occupation, and level of education, and for parents of twins in the cognition study information regarding handedness is also obtained.

The mail and phone assessment battery consists of a questionnaire, psychophysical tests (taste and smell), and measures of reading ability. The primary assessment in the questionnaire was health and wellbeing as assessed by the SPHERE, which has been shown to be a useful self-report measure of psychopathology in adults (Hadzi-Pavlovic et al., 1997; Hickie et al., 2001), coupled with measures of leisure (Passmore \& French, 2000), social interactions, and personality (NEO PI-R: particpants $17+$ years) (Costa \& McCrae, 1992). Other measures included laterality (standard questions on handedness, Annett, 1970; as well as foot and eye preference), asthma and allergies, author recognition (a measure of how widely a person reads that may be associated with reading ability), and attitudes towards parenting (for those 17 and over), as well as standard demographic questions. The self-administered psychophysical tests included the Brief Smell Identification Test (BSIT: a standardised scratch and sniff test of odour identification) (Doty, Shaman, \& Dann, 1984), the National Geographic Smell Survey (Wysocki \& Gilbert, 1989), a taste test for the sensitivity to the bitter tasting compound 6-n-propyl-2-thiouracil (PROP), and a 10-compound taste test comprising both sweet and bitter compounds, with each compound being tested twice (developed by Monell Chemical Senses Center, Philadelphia, USA). The reading measures included reading and spelling tests of nonwords, regular words and irregular words, a phonetic spelling test and a test of word meanings (developed by the Macquarie University group; e.g., Castles \& Coltheart, 1993; Castles, Datta, Gayan, \& Olson, 1998).

\section{Blood collection}

At the end of each testing session, a 40-ml blood sample is collected from participants and parents. On those occasions when parents do not accompany the twins, a blood kit containing labelled tubes, a consent form, and instructions for the phlebotomist, is sent to the parent by courier. The blood is then sent back to QIMR in a prepaid courier bag provided. The first time a blood sample is collected, $28 \mathrm{ml}$ is assigned for DNA isolation, $4 \mathrm{ml}$ for blood group typing, and $4 \mathrm{ml}$ each for haematological and biochemical analysis. For subsequent samples, blood group typing is not required and therefore $30 \mathrm{ml}$ is collected for DNA, and $5 \mathrm{ml}$ each for the haematological and biochemical analyses. Blood from parents is used primarily for DNA isolation, but also for blood group typing in the first instance. For those participants who do not wish to give a blood sample, three buccal cell samples are collected. The blood protocol has been in use since June 1998. Prior to this, a smaller sample of blood was collected for DNA isolation. Blood samples have been collected from $>90 \%$ of participants.

For all same-sex pairs, zygosity is established objectively by typing nine independent DNA microsatellite polymorphisms (polymorphism information 
content $[\mathrm{PIC}]>0.7)$ by polymerase chain reaction (PCR). These results are cross-checked with blood group results (ABO, MNS, and Rh; the blood typing being provided by the Australian Red Cross Blood Service, Brisbane), and phenotypic data (hair, skin and eye colour), giving an overall probability of correct zygosity assignment of $>99.9 \%$.

\section{Participant remuneration and communication}

Remuneration varies from study to study. In the early years of the mole study, each twin was given a "Twin Mole study" t-shirt but more recently a movie pass is given to the 12-year-olds, and at 14 they receive $\$ 10$ for taking part in the binocular rivalry study. In the cognition study a $\$ 20-\$ 30$ (performance-dependent) honorarium is given, and participants are sent two mementos of the study: a colour brain map showing their brain activity, and a picture of themselves with the electrode cap on their head. Similarly in the pelvic floor study an honorarium of $\$ 100$ in the form of a Coles/Myer voucher is offered, and in the mail study a double movie pass is sent out following completion. In addition to these honoraria, all same-sex twins are informed via letter (2-3 months after giving a blood sample) of their zygosity, and both twins and siblings are informed of their blood type.

There have also been several newsletters, in various forms, that have been mailed to the twins. This provides direct feedback to the families, and supplements the verbal feedback on study progress that is given by the research assistants at return visits. Also copies of our scientific papers and other relevant literature are available for families to peruse during their visit and when requested (e.g., for school projects); and there are several posters from various scientific meetings on the walls in the testing rooms that can be viewed. The close proximity of the testing rooms to the Genetic Epidemiology Unit also affords the opportunity for $\mathrm{PhD}$ students and research staff to be called in to answer the more scientific questions. This large amount of face-to-face contact with the families is in contrast to the more usual questionnaire-based twin studies.

\section{Sample characteristics}

Table III shows a breakdown of the number of individuals who have registered for either or both of the mole and cognition studies. As of March 2003 this consisted of 1324 families, including 1272 families each with one pair of twins, 19 families each with two pairs of twins, 32 families each with triplets, and one family with quadruplets. In addition, a large number $(n=1179)$ of singleton siblings of twins/multiples registered, along with their twin siblings. For each category (twins, multiples, singletons, and parents), there are approximately an equal number of male and female registrants. The percentage of inactive registrants, those who registered but who are now either no longer interested in participating, unable to be traced, or are deceased, is low, and ranges from $0 \%$ for multiples to $10 \%$ for fathers. In the lower half of Table III the number of twin pairs for each zygosity group is listed, this shows a 40:60 ratio of identical to nonidentical twin pairs.

Although there is a wide age range for the twins (5-28 years), 52\% are aged between 18 and 23 (year of birth $1980-1985$ ) as a result of the two major recruitments (1992 for 10-12-year-olds and 1998 for 13-16-year-olds). A further 35\% are aged between 11 and 17 (year of birth 1986-1992). Singleton siblings of the twins also vary in age $(1-38$ years), but $68 \%$ are currently aged between 11 and

Table III No. individuals (twins, multiples, singletons and parents) and no. twin pairs (MZ, DZ, DZ opposite sex, and unknown zygosity) registered (as of March 2003)

\begin{tabular}{lccr}
\hline & $\begin{array}{c}\text { Male } \\
\text { (\% inactive) }\end{array}$ & $\begin{array}{c}\text { Female } \\
n(\% \text { inactive })\end{array}$ & Total \\
\hline Twins $^{\mathrm{a}}$ & $1325(4)$ & $1295(4)$ & 2620 \\
Multiples $^{\mathrm{b}}$ & $45(0)$ & $55(0)$ & 100 \\
Singletons & $600(2)$ & $579(2)$ & 2642 \\
Parents & $1318(10)$ & $1324(6)$ & 5641 \\
Total & 3288 & 3253 & 534 \\
No. twin pairs & 266 & 268 & 418 \\
MZ & 219 & 199 & 369 \\
DZ & - & - & Total no. twin pairs 1344 \\
DZ opposite sex & - & & 23 \\
Unknown zygosity & & &
\end{tabular}

Note: ${ }^{a} 19$ families with two pairs of twins; ${ }^{b} 32$ families with triplets, one family with quadruplets; ${ }^{c}$ triplets counted as a twin pair and a sibling, quadruplets as two twin pairs. 
Table IV No. participants (and nonparticipants) in studies (as of March 2003).

\begin{tabular}{|c|c|c|c|c|}
\hline & Mole (Age 12) ${ }^{\mathrm{a}}$ & Cognition (Age 16) & Pelvic floor (Age 18+) & Mail and phone (Age 10-25) \\
\hline \multicolumn{5}{|l|}{ Participants } \\
\hline Male: Twins ${ }^{c}$ & 695 & 515 & - & 536 \\
\hline Siblings & 175 & 51 & - & 168 \\
\hline Female: Twins ${ }^{c}$ & 701 & 553 & 142 & 545 \\
\hline Siblings & 187 & 65 & 9 & 175 \\
\hline Total & 1758 & 1184 & 151 & 1424 \\
\hline \multicolumn{5}{|c|}{ Twin pairs by zygosity ${ }^{\mathrm{d}}$} \\
\hline $\mathrm{MZ}$ male & 127 & 128 & - & 62 \\
\hline MZ female & 135 & 115 & 46 & 75 \\
\hline $\mathrm{DZ}$ male & 121 & 74 & - & 102 \\
\hline DZ female & 116 & 68 & 25 & 92 \\
\hline DZ opposite sex & 199 & 149 & - & 177 \\
\hline Total & 698 & 534 & 71 & 508 \\
\hline \multicolumn{5}{|c|}{ Nonparticipants $(\%)^{\mathrm{e}}$} \\
\hline Exclusions & - & 6.0 & 11.0 & - \\
\hline Refusals & 4.0 & 11.0 & 35.0 & 7.0 \\
\hline Lost contacts & 0.8 & 5.0 & 8.0 & 2.0 \\
\hline Interstate/overseas & 0.2 & 6.0 & 10.0 & 6.0 \\
\hline
\end{tabular}

Note: $\mathrm{MZ}=$ monozygotic; $\mathrm{DZ}=$ dizygotic.

${ }^{\mathrm{a}}$ No. who participate at age 12 but not at age 14 is $<0.5 \%$; ${ }^{\mathrm{b}}$ no. calculated for reading component of study; ${ }^{\mathrm{c}}$ triplets considered as a twin pair and one sibling (no quadruplets have participated); ${ }^{\mathrm{d}}$ no. pairs where both co-twins have participated; ${ }^{\mathrm{t}}$ those invited to participate but were excluded on screening, refused, could not be traced, or who now live interstate/overseas.

23 (year of birth 1980-1992) and therefore are of similar age to the twins and multiples.

The number of participants in each of the four main studies is shown in Table IV. For all studies, excluding the pelvic floor (which included women only), there were approximately equal numbers of male and female subjects. The largest study so far is the mole study, which has been going for just over 10 years and includes 1758 individuals with 698 twin pairs and 362 siblings. However, it is expected that when data collection for the mail study is completed, the number of participants in the mail study will be $>2000$ (individuals). The number of pairs in the cognition study currently stands at 534 twin pairs, but by the end of 2003 it should be well over 600 pairs. In particular, the number of $\mathrm{DZ}$ pairs will substantially increase relative to $\mathrm{MZ}$ pairs because we are now following the same protocol as we did for the mole study a few years ago, in that $\mathrm{MZ}$ twin pairs are invited to participate only if they have a nontwin sibling. This is a practical decision because an $\mathrm{MZ}$ twin pair without siblings is not informative for genetic linkage analysis. This protocol was also followed in the mail study, and explains the smaller ratio of $M Z$ to $\mathrm{DZ}$ pairs.

The nonparticipation rate shows considerable variation across studies and is shown at the bottom of Table IV. There is a low of $5 \%$ for the mole study and a high of $64 \%$ for the pelvic floor study. This reflects several factors. In the mole study the refusal rate is low (4\%), as was expected given that only a short time has elapsed since time of registration. This is also reflected in the low percentage of lost contacts $(0.8 \%)$ and the small number of families $(0.2 \%)$ that are no longer able to participate, having moved interstate or overseas. For the cognition study the nonparticipation rate is more than 5 times that of the mole study because of an increase in refusal rate, lost contacts, and those families not able to participate because of distance, but also because a considerable number of twins was excluded due to vision impairments, a significant neurological or psychiatric history, or a previous head injury. The pelvic floor study by its intrusive nature has a much higher refusal rate $(35 \%)$ than the other studies, and there are a significant number of exclusions $(11 \%)$ because the study required neither of the co-twins to be pregnant or to have given birth. On the other hand, the low $7 \%$ refusal rate, at least thus far, for the mail study, with the three components together taking around $3 \mathrm{hr}$ of an individual's time, is astonishing, but indicative of the willingness and generosity of spirit of this adolescent/young adult twin sample.

A breakdown of the number of families who had two or more individuals participating in one or a combination of the studies showed that just under half $(45 \%)$ of the families have participated in two studies, with another quarter $(24 \%)$ having participated in three studies. Moreover, when the mail and phone study has been completed, it is predicted that the number of families participating in three studies will be $>30 \%$. This not only allows for the examination of the same phenotypes over time, but also increases the possibility of examining associa- 
tions among phenotypes collected in the different studies, for example the reading measures collected in the mail and phone study with the psychometric measures in the cognition study, the personality measures in the mail and phone study with the psychometric and electrophysiological measures of the cognition study. From a practical view, both the DNA zygosity testing and the genome-wide scan, both of which are at considerable cost to any one study ( $\$ 80$ per pair to determine zygosity, $\$ 400$ per scan for a 300-marker genome-wide scan) have to be done only once, as well as there being administrative savings.

\section{Discussion}

\section{Some research findings and highlights to date}

There are many analyses in progress on the data that have been collected. Briefly reported below are some of the main findings to date, and Figures $1-3$ show the heritability estimates for some of the major phenotypes that have been estimated thus far. Of note is that heritability estimates of many of the psychological variables are of similar magnitude to the physical measures.

\section{Mole study}

Analysis of the mole study data has primarily been undertaken by $\mathrm{Gu} \mathrm{Zhu}$. There is a very high $\mathrm{MZ}$ correlation for total naevus count (TNC; 0.94), which is as high as the test - retest correlation on the same individuals (0.96), and between mole counts on the left and right sides of the body in these twins (0.93). The DZ correlation (0.60) is much lower than that of $\mathrm{MZ}$ twins, suggesting major genetic

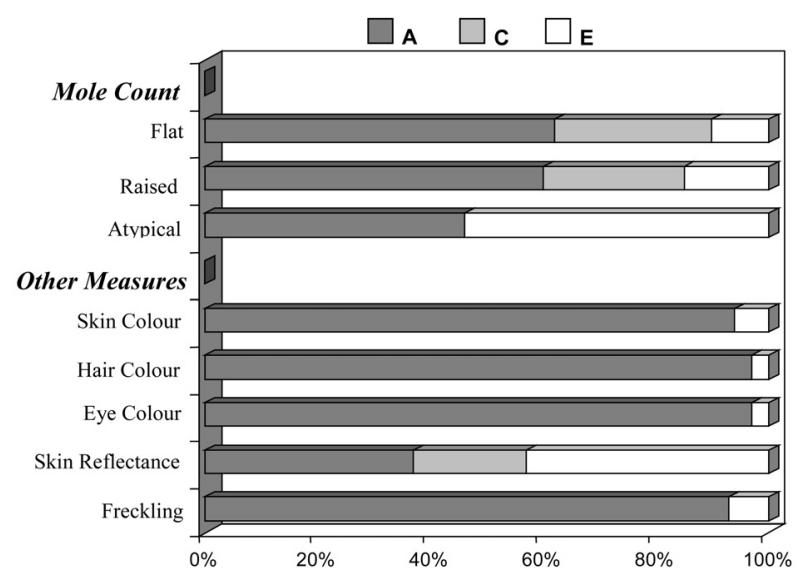

Figure 1. Heritability estimates for mole count and other risk factors for melanoma. The proportion of total variance explained by genetic (A), common (C) and unique environmental (E) factors is shown. influences on TNC in this sample. Moreover, correction for body surface area (equivalent to analysing naevus density, rather than count) makes little difference. Maximum likelihood estimation suggests that $68 \%$ of the variance in TNC is due to additive genetic factors, $26 \%$ to family environment and $6 \%$ due to unique environmental variance (McGregor et al., 1999; Zhu et al., 1999). We hypothesised that some of the genetic variance in TNC might be due to variation in the $C D K N 2 A$ (p16) gene. Analysis of linkage to a highly polymorphic marker (D9S942) located close to the $C D K N 2 A$ locus detected QTL effects accounting for $27 \%$ of variance in TNC, rising to $33 \%$ if flat but not raised moles were considered. Although the total heritability of raised moles (0.69) was much higher than that for flat moles $(0.44)$, the estimate for a QTL effect linked to $C D K N 2 A$ was zero for raised moles, suggesting that raised and flat moles have etiologies that are largely distinct (Zhu et al., 1999). We have recently completed the first stage of a genome-wide scan for moliness genes and have found a high linkage signal for flat mole count lying close to $C D K N 2 A$, in support of our earlier findings with D9S942.

\section{Cognition study}

The $\mathrm{PhD}$ thesis of Dr Michelle Luciano quantified the extent of the genetic influence on performance measures of information processing and working memory, and examined their association with psychometric IQ (Luciano, 2002). She was the first to

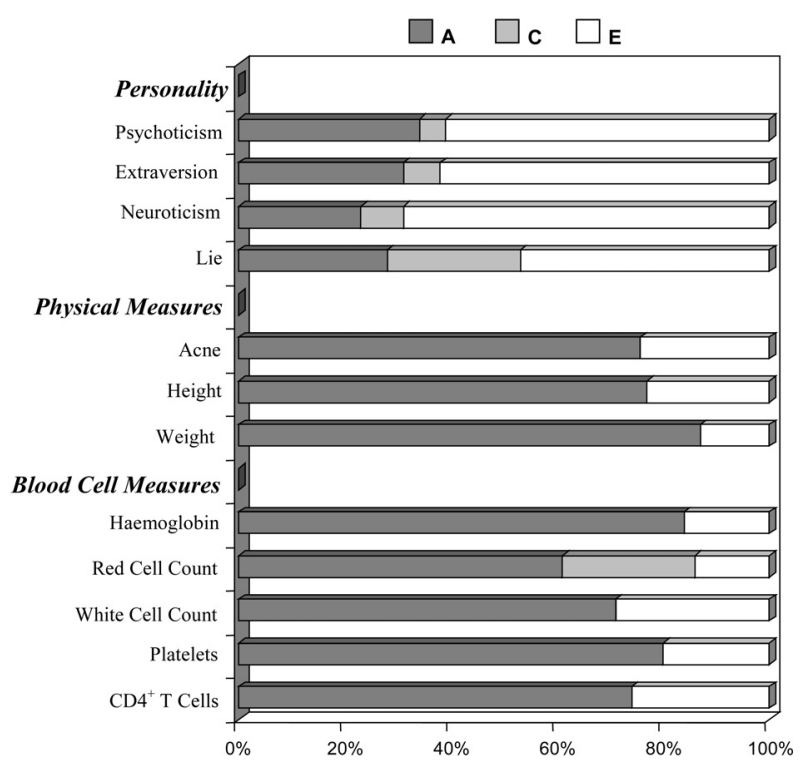

Figure 2. Heritability estimates for personality, physical and blood cell measures. The proportion of total variance explained by genetic (A), common (C) and unique environmental (E) factors is shown. 


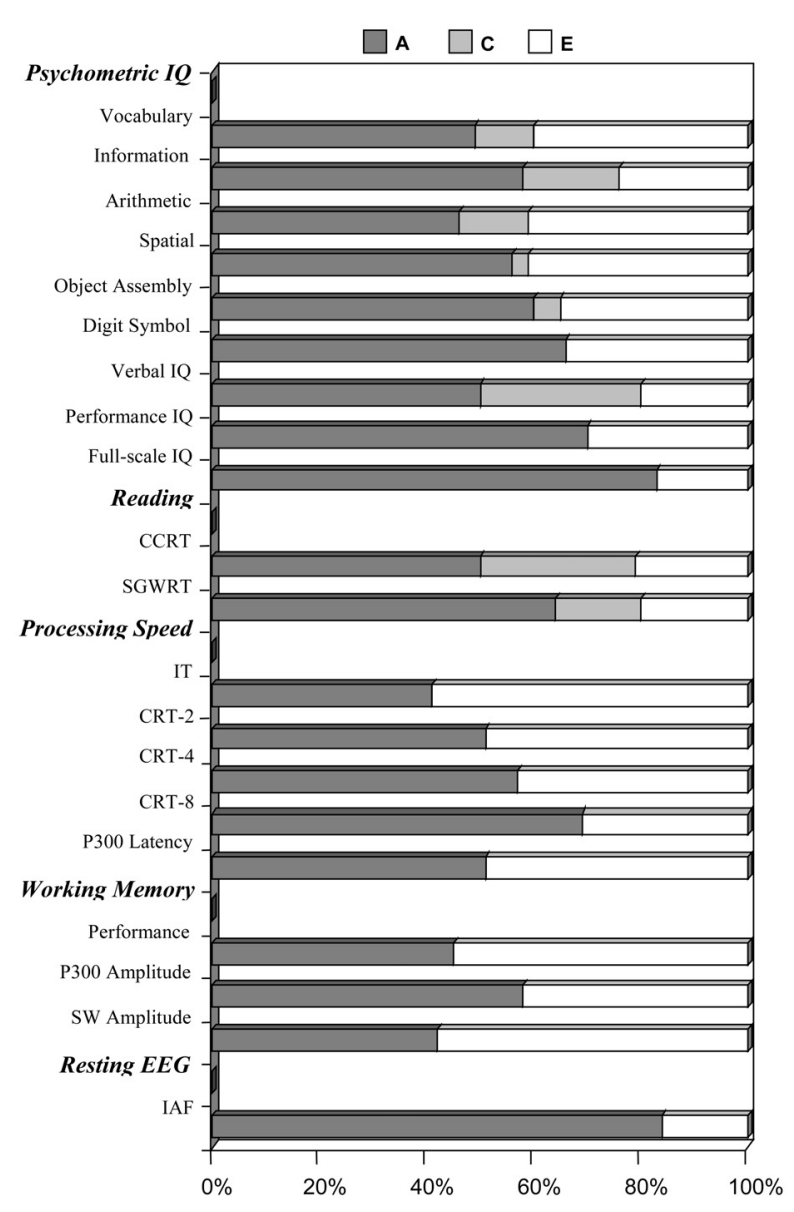

Figure 3. Heritability estimates of cognitive indices. The proportion of total variance explained by genetic (A), common (C) and unique environmental (E) factors is shown.

estimate the heritability (0.36) of IT (considered a purer measure of biological speed than measures with a motor component) (Luciano et al., 2001), and showed that the relationship for both information processing measures (IT and CRT) with IQ (phenotypic correlations of $\sim 0.30$ ) is around $90 \%$ genetically mediated (Luciano et al., 2003), highlighting the pleiotropic nature of genes influencing cognition. A multivariate genetic analysis including IQ subtest scores was found to support a factor model comprising general genetic (loadings $0.35-$ 0.66 for IQ, -0.47 and -0.24 for IT and CRT respectively) and shared environmental factors, three genetic group factors (one related to verbal subtests, one to visuospatial subtests, and the other to speed tests), specific genetic factors, plus overlapping and nonoverlapping unique environmental effects (Luciano et al., 2004a). The sources of covariation between IT, CRT, delayed response (DR) speed and accuracy (working memory performance), and IQ were further examined and support for a model including genetic and unique environmental factors indicated. The genetic structure was complex and included a general cognitive factor (loadings 0.28 $0.64)$ plus three other factors explaining the remaining genetic covariation between various speed and working memory measures with IQ. Fifty-four per cent and $25 \%$ of genetic variance was unique to respective verbal and performance IQ, and may reflect information processes not captured by our cognitive tasks (Luciano et al., 2004b).

The recently submitted $\mathrm{PhD}$ thesis of Narelle Hansell focussed on the amplitude of the eventrelated potential (ERP) SW elicited in a delayed response working memory task, which is an electrophysiological correlate of the maintenance of working memory. Her studies showed that there was a significant genetic influence on SW amplitude, but that the magnitude was regionally variable, ranging from a low of 0.08 to a high of 0.42 , and that the genetic influence on SW amplitude was generally higher at posterior than at anterior sites. Moreover, genes influencing memory processes appeared to be most active in the first $500 \mathrm{~ms}$ of the 3.5-s SW component. In addition, Hansell demonstrated that there was a strong common genetic influence on SW amplitude that was irrespective of memory load (Hansell et al., 2001), although independent genes appeared to have a small but significant influence on possible inhibitory processes occurring when memory was disrupted (Hansell et al., 2004). Building on our work with another ERP component, the P300 (Wright et al., 2001; 2002), Hansell showed that there was some association between SW amplitude and IQ that was genetically mediated. A relationship between SW amplitude and both P300 and information processing measures (CRT) was also evident and found to be genetically mediated (Hansell, 2003).

Currently, Mark Wainwright is doing his doctoral thesis centered on the reading and academic achievement phenotypes. Recently completed analyses have examined the relationship between reading tests used in neuropsychological assessment, the CCRT which assesses contextualised irregular word pronunciation, and the SGWRT which assesses word recognition, with psychometric IQ tests (Wainwright et al., 2003). Given that the CCRT is used as an estimate of premorbid IQ and that the SGWRT is used as an adjunct for assessing word reading skill in less able readers, a high phenotypic association among the reading and IQ subtests was found $(0.22-0.65)$, confirming previous studies. However, the new finding from Wainwright's work is that common genetic influences account for around $70 \%$ of the association between reading and verbal IQ subtests. This work is soon to be extended using the Macquarie reading tests that were administered as part of the mail and phone study, and which includes a larger sample (approximately 3 times). 


\section{Haematological and immunological study}

David Evans' $\mathrm{PhD}$ studies were focussed on establishing whether and to what extent genetic factors influence a wide range of haematological and immunological measures. His studies show that in contrast to the small levels of variation observed within an individual over time, blood cell measures differ remarkably between different individuals, and that a significant proportion of the variation (61$96 \%)$ is due to genetic factors. He demonstrated that although common environmental factors have a significant influence $(20-25 \%)$ on red cell count, they have only a small or nonsignificant affect on basal levels of haemoglobin, white cell count, or any T-cell (lymphocyte) counts (e.g., CD4 $+\mathrm{T}$ cell) examined (Evans et al., 1999; Evans et al., 2001) (also see Figure 3). All previous studies of this nature have been somewhat limited because of small sample size and because only a few, or selected, blood cell measures were investigated. Extending on the genetic modelling, Evans has gone on to use genetic sibpair linkage analyses, making full use of the longitudinal nature of the data (i.e., blood cell measures are collected at 12, 14,16 and some at 18 years) to increase the power, with the aim of identifying QTLs underlying the homeostatic control (e.g., mechanisms of haemopoiesis, sequestration and/or apoptosis) of blood cell measures, and which produce differences in blood cell concentrations between individuals. Promising preliminary linkages were found, for eosinophil (type of white cell) count, CD4-CD8 ratio, platelet count and total white cell count (Evans, 2003). Acknowledgement of the genes underlying the CD4-CD8 ratio, for example, might be important in developing strategies for immune cell reconstitution in HIV infection.

\section{Personality study}

Nathan Gillespie, as part of his doctoral studies that are focussing on various dimensions of personality in adolescence and adulthood, has shown that familial aggregation explains approximately $30-$ $55 \%$ of the total variance for each of the four personality dimensions of the Junior Eysenck Personality Questionnaire (JEPQ): Psychoticism, Extraversion, Neuroticism and Lie. One particular focus has been in establishing whether during adolescence there are changes in the magnitude of genetic and environmental effects measured over time, and whether the same genetic and environmental influences operate throughout time. Although there appears to be high genetic continuity across time in adult personality, at least with respect to Neuroticism and Extraversion, much less is known of the genetic and environmental continuity in adolescent personality. Using genetic simplex modelling and personality scores obtained at 12,14 and 16 years, Gillespie's analyses so far have found that the proportions of additive genetic variance underlying the four personality dimensions are stable over time, and that the genetic effects observed at age 12 are largely continuous at ages 14 and 16 . Figure 2 shows the heritability estimates of the four personality dimensions at age 12 .

\section{Laterality and fingerprint studies}

As part of her doctoral studies, Sarah Medland has been studying the laterality data collected. Although many previous studies have found familial aggregation for handedness, the source of this aggregation has remained a subject of debate (Sicotte, Woods, \& Mazziotta, 1999). Medland's analyses have found that genetic factors explain approximately $27 \%$ of the variation in handedness classification based on throwing; a measure that is not subject to high levels of social influence (Medland et al., 2003). Future work using the foot and eye preference data will determine whether these lateralities show the same pattern of results, and determine whether the significant covariation between these measures is due to a common genetic factor. In addition, Medland's preliminary analyses of the fingerprint data have shown high heritability of total ridge count, with approximately $95 \%$ of variation in this trait being explained by additive genetic factors, and consistent with previous studies (Holt, 1968). Medland is currently in the process of searching for QTLs for this highly genetic trait.

\section{Future work}

A major focus of future work will be the use of combined linkage and association techniques to narrow down the loci of interest and to pin down some of the key genes influencing a number of the main traits, together with a series of multivariate analyses to capture the genetic covariation among various phenotypes. In addition, we hope to be able to extend the phenotypic collection to include, for example, structural and functional measures of the human brain as assessed by magnetic resonance imaging. Moreover, in the long term, having recruited the sample when they were in adolescence, there is the potential to assess them again as adults, and perhaps later in life. Future potential collaborations for follow-up studies or using existing data are welcomed, as are postgraduate students who are interested in research into human individual differences. 


\section{Acknowledgements}

These studies have been supported from multiple sources: National Health and Medical Research Council (901061, 950998, 241944), Queensland Cancer Fund, Australian Research Council (A79600334, A79801419, A79906588, DP0212016) Human Frontiers Science Program (RG0154/1998-B) and Beyond Blue. We acknowledge the work and support of our collaborators and students, without which this work would not have been possible. We also are greatly appreciative of the assistance of our long-serving research nurses Ann Eldridge and Marlene Grace, project cocoordinators Alison MacKenzie and Romana Leisser, as well as many other research assistants and support staff in the Genetic Epidemiology Unit at QIMR. Thanks go to the Education Board and school principals and staff for help with contacting twins. Finally, we warmly thank the twins and their family members for their continued support, generosity of time, and for their interest in this research.

\section{References}

Almasy, L., \& Blangero, J. (2001). Endophenotypes as quantitative risk factors for psychiatric disease: Rationale and study design. American fournal of Human Genetics, 105, $42-44$.

Annett, M. (1970). A classification of hand preference by association analysis. British fournal of Psychology, 61, 303-321.

Beardsall, L., \& Huppert, F. A. (1994). Improvement in NART word reading in demented and normal older persons using the Cambridge Contextual Reading Test. Fournal of Clinical and Experimental Neuropsychology, 16, 232-242.

Berlanga, C., Heinze, G., Torres, M., Apiquian, R., \& Caballero, A. (1999). Personality and clinical predictors of recurrence of depression. Psychiatric Services, 50, 376-380.

Boomsma, D., Busjahn, A., \& Peltonen, L. (2002). Classical twin studies and beyond. Nature Review Genetics, 3, 872-882.

Boomsma, D. I., Anokhin, A., \& de Geus, E. J. C. (1997). Genetics of electrophysiology: Linking genes, brain and behavior. Current Directions in Psychological Science, 6, 106-110.

Castles, A., \& Coltheart, M. (1993). Varieties of developmental dyslexia. Cognition, 47, 149-180.

Castles, A., Datta, H., Gayan, J., \& Olson, R. K. (1998). Varieties of developmental reading disorder: Genetic and environmental influences. Fournal of Experimental Child Psychology, 72, 73-94.

Consortium, S. (2002). A genomewide scan identifies two novel loci involved in specific language impairment. American fournal of Human Genetics, 70, 384-398.

Costa, P. T., \& McCrae, R. R. (1992). Revised NEO Personality Inventory (NEO PI-R) and NEO Five-Factor Inventory (NEOFFI) professional manual. Odessa, FL: Psychological Assessment Resources.

Dietz, H. P., Herbison, P., \& Clarke, B. (2001). The predictive value of hypermobility and urethral closure pressure in the diagnosis of female stress urinary incontinence. Neurourology and Urodynamics, 20, 490-491.

Doty, R. L., Sharman, P., \& Dann, M. S. (1984). Development of the University of Pennsylvania Smell Identification test: A standardized microencapsulated test of olfactory function. Physiology and Behaviour, 32, 489-502.
Drayna, D., Coon, H., Kim, U-K., Elsner, T., Cromer, K., Otterud, B., et al. (2003). Genetic analysis of a complex trait in the Utah Genetic Reference Project: A major locus for taste ability on chromosome $7 \mathrm{q}$ and a secondary locus of chromosome 16p. Human Genetics, 112(5-6), 567-572.

Evans, D. M. (2003). The genetics of blood cell concentrations. Unpublished doctoral dissertation, University of Queensland, Brisbane, Queensland, Australia

Evans, D. M., Frazer, I. H., Boomsma, D. I., \& Martin, N. G. (2001). Developmental genetics of red cell indices during puberty: A longitudinal twin study. International fournal of Human Genetics, 1, 41-53.

Evans, D. M., Frazer, I. H., \& Martin, N. G. (1999). Genetics and environmental causes of variation in basal levels of blood cells. Twin Research, 2, 250-257.

Evans, D. M., Gillespie, N. A., \& Martin, N. G. (2002). Biometrical genetics. Biological Psychology, 61, 33-51.

Eysenck, H. J., \& Eysenck, S. B. G. (1975). Manual for the Eysenck Personality Questionnaire. London: Hodder and Stoughton.

Finkel, D., Pedersen, N. L., \& Larsson, M. (2001). Olfactory functioning and cognitive abilities: A Twin study. Fournal of Gerontology: Psychological Sciences, 56B, 226-233.

Fisher, S. E., Francks, C., Marlow, A. J., MacPhie, I. L., Newbury, D. F., Cordon, L. R., et al. (2002). Independent genome-wide scans identify a chromosome 18 quantitativetrait locus influencing dyslexia. Nature Genetics, 30, 86-91.

Gershuny, B. S., \& Sher, K. J. (1998). The relation between personality and anxiety: Findings from a 3-year prospective study. Fournal of Abnormal Psychology, 107, 252-262.

Green, A. C., \& Swerdlow, A. J. (1989). Epidemiology of melanocytic nevi. Epidemiologic Reviews, 11, 204-221.

Guo, S.-W., \& Reed, D. (2001). The genetics of phenylthiocrabamide perception. Annuals of Human Biology, 28, 111-142.

Hansell, N. K. (2003). A genetic investigation of brain function and cognitive ability. Unpublished doctoral dissertation, University of Queensland, Brisbane, Queensland, Australia.

Hansell, N. K., Wright, M. J., Geffen, G. M., Geffen, L. B., Smith, G. A., \& Martin, N. G. (2001). Genetic influences on ERP slow wave measures of working memory in adolescent twins. Behavior Genetics, 31, 603-614.

Hansell, N. K., Wright, M. J., Geffen, G. M., Geffen, L. B., \& Martin, N. G. (2004). Genetic influence on cognitive processes associated with distraction: An event-related potential study of the slow wave. Australian fournal of Psychology, 56, 89-98.

Hadzi-Pavlovic, D., Hickie, I. B., \& Hooker, A. W., (1997). The IFI: Some neurasthenia related scales (Report No. TR-97-001). Sydney: Academic Department of Psychiatry, St George Hospital.

Hickie, I. B., Davenport, T. A., Hadzi-Pavlovic, D., Koschera, A., Naismith, S. L., Scott, E. M., et al. (2001). Development of a simple screening tool for common mental disorders in general practice. Medical fournal of Australia, 175, S10-S17.

Holt, S. B. (1968). The genetics of dermal ridges. Springfield: Charles C Thomas.

Hopper, J. L. (2002). The Australian Twin Registry. Twin Research, 5, 329-336.

Jackson, D. N. (1998). Multidimensional Aptitude Battery II. Port Huron, MI: Sigma Assessment Systems.

Knoblauch, H., Muller-Myhsok, B., Busjahn, A., Ben Avi, L., Bahring, S., Baron, H., et al. (2000). A cholesterol-lowering gene maps to chromosome 13q. American Fournal of Human Genetics, 66, $157-166$.

Luciano, M. (2002). An information processing approach to the genetic study of psychometric intelligence: Links between processing speed, working memory and IQ. Unpublished doctoral dissertation, University of Queensland, Brisbane, Queensland, Australia. 
Luciano, M., Smith, G. A., Wright, M. J., Geffen, G. M., Geffen, L. B., \& Martin, N. G. (2001). On the heritability of inspection time and its covariance with IQ: A twin study. Intelligence, 29, $443-457$.

Luciano, M., Wright, M. J., Smith, G. A., Geffen, G. M., Geffen, L. B., \& Martin, N. G. (2003). Genetic covariance between processing speed and IQ. In R. Plomin, J. DeFries, P. McGuffin, \& I. Craig (Eds.), Behavior genetics in the postgenomic era (pp. 163-182). Washington, DC: American Psychological Association.

Luciano, M., Wright, M. J., Geffen, G. M., Geffen, L. B., Smith, G. A., \& Martin, N. G. (2004a). A genetic investigation of the covariation among inspection time, choice reaction time and IQ subtest scores. Behavior Genetics, 34, 41-50.

Luciano, M., Wright, M. J., Geffen, G. M., Geffen, L. B., Smith, G. A., \& Martin, N. G. (2004b). Multivariate genetic analysis of cognitive abilities in an adolescent twin sample. Australian Fournal of Psychology, 56, 79-88.

MacLennan, A. H., Taylor, A. W., Wilson, D. H., \& Wilson, P. D. (2000). The prevalence of pelvic floor disorders and their relationship to gender, age, parity and mode of delivery. British Fournal of Obstetrics and Gynaecology, 107, 1460-1470.

Martin, N. G., Boomsma, D. I., \& Machin, G. A. (1997). A twinpronged attack on complex traits. Nature Genetics, 17, $387-$ 392.

McGregor, B., Pfitzner, J., Zhu, G., Aitken, J. F., Green, A. C., \& Martin, N. G. (1999). Genetic and environmental contributions to size, colour, shape and other characteristics of melanocytic naevi in a sample of adolescent twins. Genetic Epidemiology, 16, 40-53.

Medland, S. E., Wright, M. J., Geffen, G. M., Hay, D. A., Levy, F., Martin, N. G., et al. (2003). Special twin environments and their effects on the handedness of twins and their siblings. Twin Research, 6, 119-130.

Miller, S. M., Liu, G. B., Ngo, T. T., Hooper, G., Riek, S., Carson, R. G., et al. (2000). Interhemispheric switching mediates perceptual rivalry. Current Biology, 10, 383-339.

Neale, M. C., \& Cardon, L. R. (1992). Methodology for genetic studies of twins and families, NATO ASI Series D: Behavioural and Social Sciences (Vol. 67). Dordrecht: Kluwer Academic.

Passmore, A., \& French, D. (2000). A model of leisure and mental health in Australian adolescents. Behaviour Change, 17, 208220 .
Plomin, R., DeFries, J. C., McClearn, G. E., \& Rutter, M. (1997). Behavioral genetics (4th ed.). New York: Freeman.

Posthuma, D., \& Boomsma, D. I. (2000). A note on the statistical power in extended twin designs. Behavior Genetics, 30, $147-$ 158.

Segal, N. L., Topolski, T. D., Wilson, S. M., Brown, K. W., \& Araki, L. (1995). Twin analysis of odor identification and perception. Physiology and Behavior, 57, 605-609.

Sicotte, N., Woods, R., \& Mazziotta, J. (1999). Handedness in twins: A meta-analysis. Laterality, 4, 265-286.

Swerdlow, A. J., \& Green, A. C. (1987). Melanocytic naevi and melanoma: An epidemiological perspective. British fournal of Dermatology, 117, 137-146.

Wainwright, M. A., Wright, M. J., Luciano, M., Geffen, G. M., Geffen, L. B., \& Martin, N. G. (2003) Genetic and environmental sources of covariance between reading tests used in neuropsychological assessment and IQ subtests. Behavior Genetics, 34, 365-376.

Wechsler, D. (1981). Wechsler Adult Intelligence Scale-Revised manual. New York: Psychological Corporation.

Wright, M. J., De Geus, E. C., Ando, J., Luciano, M., Posthuma, D., Ono, Y., et al. (2001). Genetics of cognition: Outline of a collaborative twin study. Twin Research, 4, 48-56.

Wright, M. J., Hansell, N. K., Geffen, G. M., Geffen, L. B., Smith, G. A., \& Martin, N. G. (2001). Psychophysiological phenotypes of cognition: The P3 component. Behavior Genetics, 31, 555-565.

Wright, M. J., Luciano, M., Hansell, N. K., Geffen, G. M., Geffen, L. B., \& Martin, N. G. (2002). Genetic sources of covariation among $\mathrm{P} 3(00)$ and online performance in a delayed response working memory task. Biological Psychology, 61, 183202.

Wysocki, C. J., \& Gilbert, A. N. (1989). The National Geographic smell survey: Effects of age are heterogeneous. Annals of the New York Academy of Sciences, 561, 12-28.

Zhu, G., Duffy, D. L., Eldridge, A., Grace, M., Mayne, C., O'Gorman, L. O., et al. (1999). A major quantitative-trait locus for mole density is linked to the familial melanoma gene CDKN2A: A maximum-likelihood combined linkage and association analysis in twins and their sibs. American fournal of Human Genetics, 65, 483-492. 